Vol.61: e18160650, 2018 http://dx.doi.org/10.190/1678-4324-2018160650 ISSN 1678-4324 Online Edition
BRAZILIAN ARCHIVES OF BIOLOGY AND TECHNOLOGY

\title{
Diversity of Plant Species in The Steel City of Odisha, India: Ethnobotany and Implications for Conservation of Urban Bio-Resources
}

\author{
Sanjeet Kumar ${ }^{1}$, Gitishree Das ${ }^{2}$, Han-Seung Shin ${ }^{3}$, Pradeep Kumar ${ }^{4}$ and Jayanta Kumar Patra ${ }^{2}$. \\ ${ }^{1}$ Ambika Prasad Research Foundation, Cuttack, Odisha, India; ${ }^{2}$ Research Institute of Biotechnology \& Medical \\ Converged Science, Dongguk University-Seoul, Ilsandong-gu, Goyang-si, Gyeonggi, Republic of Korea; \\ ${ }^{3}$ Department of Food Science and Biotechnology, Dongguk University-Seoul, Ilsandong-gu, Goyang, Republic of \\ Korea; ${ }^{4}$ Department of Forestry, North Eastern Regional Institute of Science and Technology (Deemed University), \\ Nirjuli-791109, Arunachal Pradesh, India.
}

\begin{abstract}
The vegetation of the Steel City (Rourkela) of Odisha, India has high ethnobotanical values by virtue of its rich floral diversity. People in the urban area are highly dependent on the plants available in and around the city for their primary needs. The present study highlights the use of local flora, explore, identification, ethnobotany and conservation of wild and cultivated plant species in the city of Rourkela, Odisha, India. It also pays heed to the proper utilization of urban flora as a way of highlighting its ethnopharmacological importance. A field survey was conducted to collect information about floral diversity in and around the city. Data on the use of plants was collected with a semi-structured questionnaire and from the peer-reviewed literature. A total of 154 plant species, belonging to 128 genera and 55 families, were identified, along with their botanical name, vernacular name, family and habitat. Of these plant species, 53 are medicinal, 43 are ornamental, and 33 are edible, while 23 are weeds. Paderia foetida and Saraca asoka fall into the RET (rare, endangered and threatened) group and are very effective against various diseases. Traditional uses of local plants in an urban area like Rourkela are very interesting. This shows that, not only rural and tribal areas are rich in useful bio-resources but so are urban or semi-urban areas. The documentation of all useful flora with ethnomedicinal potential is helpful in conserving plant biodiversity as well as in environmental studies along with potential applications in drug discovery and oriental medicine.
\end{abstract}

Key words: conservation; ethnobotany; ethnopharmacology; floral diversity

* Author(s) for correspondence: jkpatra@dongguk.edu 


\section{INTRODUCTION}

Human beings cannot survive on this planet without floral diversity which provides resources for life in the form of food, shelter, clothing and, more essentially, atmospheric oxygen for breathing [1]. Floral diversity provides food, medicine, and fodder and has paramount economic and socio-cultural worth throughout the world [2]. Floral diversity is the prime component of biological diversity, represented by medicinal, edible, economic, and socio-cultural plants as well as by diverse other floras that are beneficial to humans and the environment $[3,4]$. These plants are either wild or cultivated [5]. Of all these plants, edible and medicinal plants in particular play an important role in human life [6]. They provide primary food and medicine to local communities [7,8]. Even in the modern era, most rural and tribal communities who live close to the forest depend on plants for their daily food and healthcare needs $[9,10]$. The medicinal uses of plants and plant parts are ancient practices [11,12]. Ancient Indian literature indicates that therapeutic uses of plants have been practiced since as long ago as 5000-4000 B.C. [13].

Floral diversity is directly proportional to chemical diversity (bioactive compounds), as reflected in the traditional knowledge of the aboriginal peoples and this area of science is known as "ethnobotany," or sometimes ethnopharmacology [14, 15].

Ethno-medicinal studies offer immense scope and opportunities for biodiversity conservation and sustainable development of local communities around the world

[16]. Wild plants are used in the form of fruits, tubers, flowers, leafy vegetables etc. for food and medical purposes [17]. The World Health Organization (WHO) has estimated that as much as $80 \%$ of the world's population depends on traditional medicine for their primary healthcare requirements [18]. Traditional medicine is a combination of both knowledge and practices, whether explainable or not; it is used in disease diagnosis and treatment, prevention and elimination of physical, mental, or social imbalance, and relies exclusively on practical experience and observations that are transferred from generation to generation by individuals [19]. Today, there is an increasing desire to reveal the role of ethno-botanical knowledge by capturing centuries-old traditional folk knowledge from elderly people as well as by searching for new plants species of medicinal and economic importance [20].

The medicinal potential of plant species and parts that are used for the preparation and administration of various drugs vary with climate and environmental conditions

[21]. However, the knowledge of herbal medicine is gradually dying out, although some traditional herbal healers around the world continue to practice the art of herbal healing effectively [22]. Floral diversity is not only rich in or near the forest or rural areas, but it is also rich in small patches of urban areas, mainly campuses of educational institutions and other government bureaus. These areas play a vital role in balancing pollution and other environmental factors in urban environments. Keeping this in mind, an attempt has therefore been made to document the useful flora in and around the Steel City (Rourkela) of Odisha State in India and its ethnobotanical potential.

\section{MATERIALS AND METHODS}

\section{Study area}

Rourkela is known as the Steel City of Odisha State in India. It is unique in floral diversity in an urban environment. It is situated between $22^{\circ} 14^{\prime} 57^{\prime \prime} \mathrm{N}$ and $82^{\circ} 54^{\prime}$ $58^{\prime \prime} \mathrm{E}$ (Plate 1). In recent years, the natural wild flora of the city has been adversely affected by the spread of concrete forests and other anthropogenic activities like mining industries and factory construction. 
Diversity, ethnobotany of plant species of Steel City, India

The average height of the study area is about $219 \mathrm{~m}$ above mean sea level. Geographically, it is a land mass of red and laterite soils and quite rich in minerals, particularly iron ore (Plate 1). The study area is very close to Vedvyas, where the Koel and Sankha rivers meet and form a single river named Brahmani. The city enjoys a tropical climate and receives high rainfall during the Southeast monsoon and the retreating Northeast monsoon. Average annual rainfall is about 160 to 200 $\mathrm{cm}$. It has semi-evergreen or tropical dry deciduous forest [23-26].

\section{Enumeration of plant species and their ethnobotany}

Field studies were carried out in different seasons of the year of 2014-2015 with the aim of enumerating the floral wealth in the city area of Rourkela by means of the Bentham \& Hooker system followed by Christian and Brigitte [27]. Seasonal variations and the frequency of occurrence of plants were noted during the field study. During field visits, surveys were undertaken in different locations, namely, waste land, bare land, play grounds, road sides, grass land, gardens, and plant species were recorded. Common and ethnobotanical uses of different plant species were recorded from people inhabiting the city and surrounding areas through a set of questionnaires in the form of a passport data form. Plant species were identified by the first author on the basis of flora books [28,29] and published articles [30]. Each species was tabulated together with its botanical name, local/common name, habit, nature and type of the plant species.

\section{RESULTS}

The field survey showed that the city of Rourkela, Odisha, India is blessed with a large number of beneficial plants with medicinal importance. During the present study, 154 plant species in 128 genera and 55 families were recorded (Fig. 1). Survey results demonstrated the wide diversity of plant species in the city. Taxa included monocotyledonous and diocotyledons. Asteraceae contributed the largest number with 12 species, followed by apocynaceae and caselpinaceae with 9 species, euphorbiaceae contributed 7 species, and fabaceae and malvaceae contributed 6 species each. Of the identified species, most were trees (45) and herbs (49); shrubs were also common (28). Climbers were rare (20) and grasses were the least common (5) (Fig. 2). The most common usage classifications of taxa were medicinal (53) (Table 1), ornamental (43) (Table 3), edible (33) (Table 2), common weed (23), timber (14), and cultural (2) (Fig. 3). The study indicates that the largest number of medicinal plants belong to tree group (Fig. 2, 3 and 4).

The most common medicinal plants are Triumfetta pentandra (Plate 2.9), Leonotis nepetifolia (Plate 2.5), Passiflora foetida (Plate 2.3, Plate 2.8), Borehivia diffusa, Terminalia bellarica (Plate 2.4), Paderia foetida, Saraca asoka and Abutilon indicum; edible plants are Dioscorea bulbifera, Amaranthus spinosus, Annona reticulate (Plate 2.2), Annona squamosa, Artocarpus heterophyllus, Commelina benghalensis, Dillenia indica, Dioscorea alata, Emblica officinalis, and Mitragyana parviflora; common weeds are Pelicetum penicellatum, Ipomea carnea, Tridex procumbens, and Xanthium strumarium; common cultivated plants are Peltophorum pterocarpum, Allamanda cathartica, Bauhinia acuminate, and Canna indica. There are some plant found in this region which are categorized as RET (Plate 3). The medicinal and ethnobotanical values of the different collected plant species are provided in Table 1-3 and Fig. 1-4. 
Table 1: List of medicinal plants in the City of Rourkela, Odisha, India

\begin{tabular}{|c|c|c|c|c|c|}
\hline Botanical names & $\begin{array}{l}\text { Local } \\
\text { name(s)/ } \\
\text { Common } \\
\text { name(s) }\end{array}$ & Family & $\begin{array}{l}\text { Habit } \\
\text { at }\end{array}$ & Group(s) & $\begin{array}{l}\text { Common uses / Source(s) } \\
\text { other character(s) }\end{array}$ \\
\hline Abrus precatorius L. & Kaincho & Fabaceae & $\begin{array}{l}\text { Climb } \\
\text { er }\end{array}$ & Medicinal & $\begin{array}{l}\text { Seeds are used for [30] } \\
\text { avoiding conception } \\
\text { during menstruation }\end{array}$ \\
\hline Abutilon indicum L. & Pedi-pedika & Malvaceae & Shrub & Medicinal & $\begin{array}{l}\text { Root and bark is [31] } \\
\text { used as nervine } \\
\text { tonic }\end{array}$ \\
\hline $\begin{array}{l}\text { Aerva lanata (L.) } \\
\text { Juss. ex. Schultes }\end{array}$ & Paunsia & $\begin{array}{l}\text { Amaranthace } \\
\text { ae }\end{array}$ & Herb & Medicinal & $\begin{array}{l}\text { Whole plant is used } \\
\text { for cholera }\end{array}$ \\
\hline $\begin{array}{l}\text { Aloe vera (L.) } \\
\text { Burn.f. }\end{array}$ & Ghee-kumari & Liliaceae & Herb & Medicinal & $\begin{array}{l}\text { Leaf juice is used to } \\
\text { treat bowel }\end{array}$ \\
\hline $\begin{array}{l}\text { Alternanthera sessils } \\
\text { (L.) R.Br. ex DC. }\end{array}$ & Kolamsago & $\begin{array}{l}\text { Amaranthace } \\
\text { ae }\end{array}$ & Herb & Medicinal & $\begin{array}{l}\text { Leaves are used for } \\
\text { treatment }\end{array}$ \\
\hline & & & & & $\begin{array}{l}\text { snakebite in Tamil } \\
\text { Nadu }\end{array}$ \\
\hline $\begin{array}{l}\text { Andrographis } \\
\text { paniculata (Burm.f.) }\end{array}$ & Bhuinimbo & Acanthaceae & Herb & Medicinal & $\begin{array}{l}\text { Leaves are used to Present study } \\
\text { treat diabetes }\end{array}$ \\
\hline $\begin{array}{l}\text { Azadirachta indica } \\
\text { Ajuss. }\end{array}$ & Limba & Meliaceae & Tree & Medicinal & $\begin{array}{l}\text { Fresh leaf juice is [30] } \\
\text { used to treat } \\
\text { different types of } \\
\text { skin infections }\end{array}$ \\
\hline $\begin{array}{l}\text { Biophytum } \\
\text { sensitivum (L.) DC. }\end{array}$ & Lokachanna & Oxalidaceae & Herb & Medicinal & $\begin{array}{l}\text { Whole plant is used }[30] \\
\text { to reduce } \\
\text { inflammation pain }\end{array}$ \\
\hline Boerhavia diffusa L. & Parani sago & $\begin{array}{l}\text { Nyctaginacea } \\
\mathrm{e}\end{array}$ & Herb & Medicinal & $\begin{array}{l}\text { Roots are used as [30] } \\
\text { diuretic }\end{array}$ \\
\hline $\begin{array}{l}\text { Calotropis gigantea } \\
\mathrm{R} . \mathrm{Br} \text {. }\end{array}$ & Arko & $\begin{array}{l}\text { Asclepiadace } \\
\text { ae }\end{array}$ & Shrub & Medicinal & $\begin{array}{l}\text { Root powder is [30] } \\
\text { used against eczema }\end{array}$ \\
\hline Cassia fistula $\mathrm{L}$. & Sonari & $\begin{array}{l}\text { Caesalpinace } \\
\text { ae }\end{array}$ & Tree & Medicinal & $\begin{array}{lll}\begin{array}{l}\text { Leaves } \\
\text { against }\end{array} & \text { used Present study } \\
\text { infections } & \text { skin }\end{array}$ \\
\hline $\begin{array}{l}\text { Cassia occidentalis } \\
\text { L. }\end{array}$ & Kasinda & $\begin{array}{l}\text { Caselpinacea } \\
\text { e }\end{array}$ & Shrub & Medicinal & $\begin{array}{l}\text { Leaf } \\
\text { externally } \\
\text { to wounds }\end{array}$ \\
\hline $\begin{array}{l}\text { Cissampelos pareira } \\
\text { L. }\end{array}$ & Paru & $\begin{array}{l}\text { Menispermac } \\
\text { eae }\end{array}$ & $\begin{array}{l}\text { Climb } \\
\text { er }\end{array}$ & Medicinal & $\begin{array}{l}\text { Leaves are used for } \\
\text { asthma }\end{array}$ \\
\hline Clitoria ternatea L. & Aparajita & Fabaceae & $\begin{array}{l}\text { Climb } \\
\text { er }\end{array}$ & Medicinal & $\begin{array}{l}\text { Flowers are toxic Present study } \\
\text { and used against } \\
\text { skin infections }\end{array}$ \\
\hline Cocculus hirsutus L. & Musakani & $\begin{array}{l}\text { Menispermac } \\
\text { eae }\end{array}$ & $\begin{array}{l}\text { Climb } \\
\text { er }\end{array}$ & Medicinal & $\begin{array}{l}\text { Leaves } \quad \text { poss } \\
\text { aphrodisiac } \\
\text { property }\end{array}$ \\
\hline Datura metel L. & Duddura & Solanaceae & Shrub & Medicinal & $\begin{array}{l}\text { Leaves are used for Present study } \\
\text { skin infections }\end{array}$ \\
\hline $\begin{array}{l}\text { Desmodium } \\
\text { gangeticum (L.) DC. }\end{array}$ & Saloporni & Fabaceae & Shrub & Medicinal & $\begin{array}{l}\text { Aqueous leaf [33] } \\
\text { extract is toxic } \\
\text { against E.coli }\end{array}$ \\
\hline Eclipta prostrate L. & Bhrungaraj & Asteraceae & Herb & Medicinal & $\begin{array}{l}\text { Leaf extracts show [34] } \\
\text { good antibacterial } \\
\text { activity }\end{array}$ \\
\hline Elephantopus scaber & Tutamuli & Asteraceae & Herb & Medicinal & Whole plant is used \\
\hline
\end{tabular}


Diversity, ethnobotany of plant species of Steel City, India

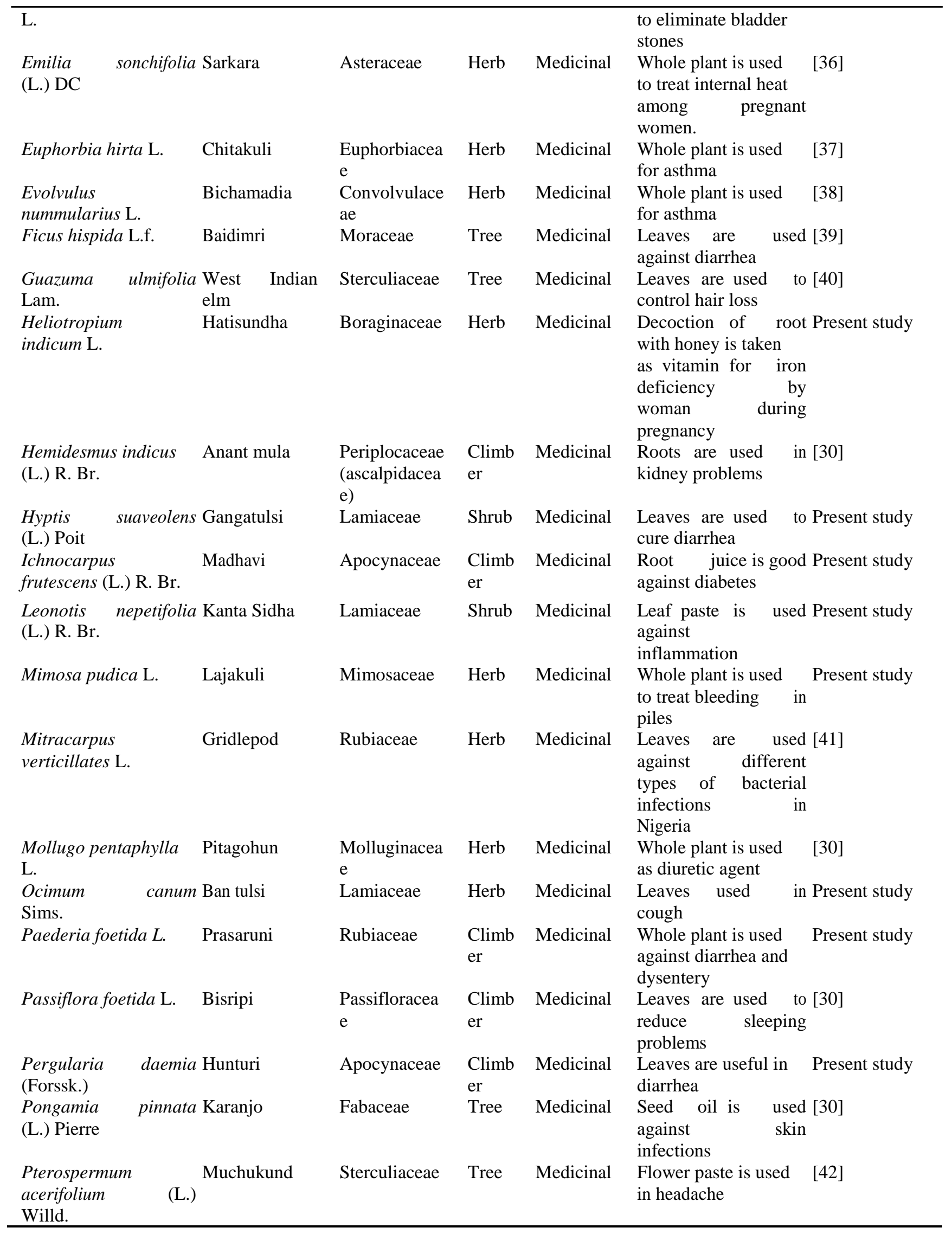




\begin{tabular}{|c|c|c|c|c|c|c|}
\hline Ricinus communis L. & Joda & $\begin{array}{l}\text { Euphorbiacea } \\
\text { e }\end{array}$ & Shrub & Medicinal & $\begin{array}{l}\text { Seed oil is used to } \\
\text { cure skin infections }\end{array}$ & Present study \\
\hline Rungia pectinata $\mathrm{L}$. & Sankh sago & Acanthaceae & Shrub & Medicinal & $\begin{array}{l}\text { Leaves are used } \\
\text { reduce swelling and } \\
\text { pain }\end{array}$ & to [43] \\
\hline \multicolumn{2}{|c|}{$\begin{array}{l}\text { Saraca asoca Oshoko } \\
\text { (Roxb.) de Wilde. }\end{array}$} & $\begin{array}{l}\text { Caesalpinace } \\
\text { ae }\end{array}$ & Tree & Medicinal & $\begin{array}{l}\text { Flowers are used in } \\
\text { painful menses }\end{array}$ & Present study \\
\hline Sida acuta Burm. f. & Bajarmuli & Malvaceae & Herb & Medicinal & $\begin{array}{l}\text { Whole plant is used } \\
\text { against dandruff }\end{array}$ & {$[30]$} \\
\hline Sida cordifolia $\mathrm{L}$. & Bisiripi & Malvaceae & Herb & Medicinal & $\begin{array}{l}\text { Leaves } \text { are used } \\
\text { nasal } \\
\text { aching }\end{array}$ & $\begin{array}{l}\text { in }[30] \\
\text { on }\end{array}$ \\
\hline \multicolumn{2}{|c|}{$\begin{array}{l}\text { Terminalia arjuna Arjuna } \\
\text { (Roxb. ex DC.) }\end{array}$} & $\begin{array}{l}\text { Combretacea } \\
\text { e }\end{array}$ & Tree & Medicinal & \multicolumn{2}{|c|}{$\begin{array}{l}\text { Bark paste is used [44] } \\
\text { in skin related } \\
\text { problems }\end{array}$} \\
\hline \multicolumn{2}{|c|}{$\begin{array}{l}\text { Terminalia bellirica Bahada } \\
\text { (Gaertn.) Roxb. }\end{array}$} & $\begin{array}{l}\text { Combretacea } \\
\text { e }\end{array}$ & Tree & Medicinal & $\begin{array}{l}\text { Bark is useful } \\
\text { leucoderma }\end{array}$ & in [44] \\
\hline $\begin{array}{l}\text { Terminalia } \\
\text { L. }\end{array}$ & catappa Desibadam & $\begin{array}{l}\text { Combretacea } \\
\text { e }\end{array}$ & Tree & Medicinal & Fruits are edible & [44] \\
\hline \multicolumn{2}{|l|}{$\begin{array}{l}\text { Terminalia } \\
\text { tomentosa Roxb. Ex } \\
\text { DC }\end{array}$} & $\begin{array}{l}\text { Combretacea } \\
\text { e }\end{array}$ & Tree & Medicinal & \multicolumn{2}{|c|}{$\begin{array}{l}\text { Bark is useful in [45] } \\
\text { ulcer }\end{array}$} \\
\hline \multicolumn{2}{|l|}{ Tribulus terrestris L. } & $\begin{array}{l}\text { Zygophyllace } \\
\text { ae }\end{array}$ & Herb & Medicinal & $\begin{array}{l}\text { Whole prant is used } \\
\text { as tonic }\end{array}$ & Present study \\
\hline $\begin{array}{l}\text { Triumfetta } \\
\text { pentandra A. Rich. }\end{array}$ & $\begin{array}{l}\text { Fivestamen } \\
\text { burbark }\end{array}$ & Tilaceae & Shrub & Medicinal & $\begin{array}{l}\text { Roots are used as a } \\
\text { cooling agent }\end{array}$ & [8] \\
\hline \multicolumn{2}{|c|}{$\begin{array}{l}\text { Verononia cinerea Badi poksunga } \\
\text { (L.) Less. }\end{array}$} & Asteraceae & Herb & Medicinal & $\begin{array}{l}\text { Whole plant is used } \\
\text { in asthma }\end{array}$ & {$[30]$} \\
\hline \multicolumn{2}{|c|}{$\begin{array}{l}\text { Wattakaka volubilis Dudhika } \\
\text { L. }\end{array}$} & $\begin{array}{l}\text { Asclepidacea } \\
\mathrm{e}\end{array}$ & $\begin{array}{l}\text { Climb } \\
\text { er }\end{array}$ & Medicinal & \multicolumn{2}{|l|}{$\begin{array}{l}\text { Leaves used } \\
\text { inflammation }\end{array}$} \\
\hline \multicolumn{2}{|c|}{$\begin{array}{l}\text { Wedelia chinensis Bhimraj } \\
\text { (Osbeck) Merr. }\end{array}$} & Asteraceae & Herb & Medicinal & \multicolumn{2}{|l|}{$\begin{array}{l}\text { Leaves are } \\
\text { against fever }\end{array}$} \\
\hline \multicolumn{2}{|c|}{$\begin{array}{l}\text { Wrightia tinctoria Pita karuna } \\
\text { (Roxb.) R. Br. }\end{array}$} & Apocynaceae & Tree & Medicinal & \multicolumn{2}{|l|}{$\begin{array}{l}\text { Leaves are used } \\
\text { tooth ache }\end{array}$} \\
\hline
\end{tabular}

Table 2: Edible plants and their common uses in the City of Rourkela, Odisha, India

\begin{tabular}{|c|c|c|c|c|c|c|c|}
\hline Botanical names & $\begin{array}{l}\text { Local } \\
\text { name }(s) / \\
\text { Common } \\
\text { name(s) }\end{array}$ & Family & Habitat & Group(s) & $\begin{array}{l}\text { Common uses } \\
\text { other character(s) }\end{array}$ & I & Source(s) \\
\hline $\begin{array}{l}\text { Aegle marmelos } \\
\text { L. }\end{array}$ & Belo & Rutaceae & Tree & Edible & $\begin{array}{l}\text { Fruits are used as a } \\
\text { cooling agent }\end{array}$ & & Present study \\
\hline Allium сера $\mathrm{L}$. & Piaja & Amaryllidaceae & Herb & Edible & $\begin{array}{l}\text { Bulbs are edible } \\
\text { vegetables }\end{array}$ & as & Present study \\
\hline $\begin{array}{l}\text { Amaranthus } \\
\text { spinosus L. }\end{array}$ & Kanta sago & Amaranthaceae & Herb & Edible & $\begin{array}{l}\text { Leaves are used } \\
\text { leafy vegetables }\end{array}$ & as & [30] \\
\hline $\begin{array}{l}\text { Amaranthus } \\
\text { viridis L. }\end{array}$ & Khoda sago & Amaranthaceae & Herb & Edible & $\begin{array}{l}\text { Leaves are used } \\
\text { leafy vegetables }\end{array}$ & as & [30] \\
\hline $\begin{array}{l}\text { Annona } \\
\text { reticulate L. }\end{array}$ & Ramphala & Annonaceae & Tree & Edible & Fruits are edible & & Present study \\
\hline $\begin{array}{l}\text { Annona } \\
\text { squamosa } \mathrm{L} .\end{array}$ & Ata & Annonaceae & Tree & Edible & Fruits are edible & & Present study \\
\hline $\begin{array}{l}\text { Artocarpus } \\
\text { heterophyllus } \\
\text { Lam. }\end{array}$ & Panasa & Moraceae & Tree & Edible & Fruits are edible & & Present study \\
\hline Basella alba $\mathrm{L}$. & Poi & Basellaceae & Climber & Edible & $\begin{array}{l}\text { Leaves are used } \\
\text { leafy vegetables }\end{array}$ & as & Present study \\
\hline
\end{tabular}


Diversity, ethnobotany of plant species of Steel City, India

\begin{tabular}{|c|c|c|c|c|c|c|}
\hline $\begin{array}{l}\text { Borassus } \\
\text { flabellifer } \mathrm{L} \text {. }\end{array}$ & Tala & Arecaceae & Palm & Edible & Jelly seeds are edible & [49] \\
\hline $\begin{array}{l}\text { Capsicum annum } \\
\text { L. }\end{array}$ & Lonka & Solanaceae & Herb & Edible & $\begin{array}{l}\text { Fruits are used as } \\
\text { spices }\end{array}$ & Present study \\
\hline Carica papaya $\mathrm{L}$. & Bhanda & Caricaceae & Shrub/tree & Edible & Fruits are edible & Present study \\
\hline Caryota urens $\mathrm{L}$. & Salapo & Arecacea & Palm & Edible & $\begin{array}{l}\text { Stem juice is used as } \\
\text { a cooling agents }\end{array}$ & Present study \\
\hline $\begin{array}{l}\text { Commelina } \\
\text { benghalensis L. }\end{array}$ & Khet papra & Commelinaceae & Herb & Edible & $\begin{array}{l}\text { Leaves are used as } \\
\text { leafy vegetables }\end{array}$ & Present study \\
\hline Dillenia indica $\mathrm{L}$. & Oao & Dilleniaceae & Tree & Edible & Fruits are edible & {$[50]$} \\
\hline $\begin{array}{l}\text { Dioscorea alata } \\
\text { L. }\end{array}$ & $\begin{array}{l}\text { Khambo } \\
\text { āalu }\end{array}$ & Dioscoreaceae & Climber & Edible & Tubers are edible & Present study \\
\hline $\begin{array}{l}\text { Dioscorea } \\
\text { bulbifera } \mathrm{L} .\end{array}$ & Pita āalu & Dioscoreaceae & Climber & Edible & $\begin{array}{ll}\text { Tubers } & \text { are edible } \\
\text { after } & \text { successive } \\
\text { boiling } & \end{array}$ & Present study \\
\hline $\begin{array}{l}\text { Dioscorea } \\
\text { hamiltonii }\end{array}$ & Suta āalu & Dioscoreaceae & Climber & Edible & Tubers are edible & Present study \\
\hline $\begin{array}{l}\text { Hook.f. } \\
\text { Dioscorea } \\
\text { oppositifolia } \text { L. }\end{array}$ & Pani aalu & Dioscoreaceae & Climber & Edible & Tubers are edible & Present study \\
\hline $\begin{array}{l}\text { Emblica } \\
\text { officinalis }\end{array}$ & Amla & Euphorbiaceae & Tree & Edible & Fruits are edible & [51] \\
\hline $\begin{array}{l}\text { Gaertn. } \\
\text { Luffa aegyptica } \\
\text { Mill. }\end{array}$ & Bān Jani & Cucurbitacea & Climber & Edible & $\begin{array}{l}\text { Fruits are used as } \\
\text { vegetables }\end{array}$ & Present study \\
\hline $\begin{array}{l}\text { Madhuca indica } \\
\text { Gmel. }\end{array}$ & Mahula & Sapotaceae & Tree & Edible & $\begin{array}{l}\text { Flowers are used in } \\
\text { making } \quad \text { country } \\
\text { liquor }\end{array}$ & Present study \\
\hline $\begin{array}{l}\text { Mangifera indica } \\
\text { L. }\end{array}$ & Amba & Anacardiaceae & Tree & Edible & $\begin{array}{l}\text { Fruits are used in } \\
\text { making pickles }\end{array}$ & Present study \\
\hline $\begin{array}{l}\text { Moringa oleifera } \\
\text { Lam }\end{array}$ & Sajana & Moringaceae & Tree & Edible & $\begin{array}{l}\text { Fruits, flowers and } \\
\text { leaves are edible as } \\
\text { vegetables }\end{array}$ & Present study \\
\hline $\begin{array}{l}\text { Murraya koenigii } \\
\text { (L.) Spreng }\end{array}$ & Bansango & Rutaceae & Herb & Edible & $\begin{array}{l}\text { Leaves are used as } \\
\text { curry leaf }\end{array}$ & Present study \\
\hline $\begin{array}{l}\text { Pithecellobium } \\
\text { dulce (Roxb.) } \\
\text { Benth. }\end{array}$ & $\begin{array}{l}\text { Simakonia, } \\
\text { Jalabi }\end{array}$ & Mimosaceae & Tree & Edible & $\begin{array}{l}\text { Fruits are edible for } \\
\text { monkeys }\end{array}$ & Present study \\
\hline $\begin{array}{l}\text { Psidium gaujava } \mathrm{l} \\
\mathrm{L} .\end{array}$ & Pijuli & Myrtaceae & Tree & Edible & Fruits are edible & Present study \\
\hline $\begin{array}{l}\text { Sesamum } \\
\text { indicum L. }\end{array}$ & Bān Rasi & Pedaliaceae & Herb & Edible & Seed oil is edible & Present study \\
\hline $\begin{array}{l}\text { Solanum } \\
\text { melongena } \mathrm{L} .\end{array}$ & Kantra & Solanaceae & Herb & Edible & $\begin{array}{l}\text { Fruits are edible as } \\
\text { vegetables }\end{array}$ & Present study \\
\hline $\begin{array}{l}\text { Solanum nigrum } \\
\text { L. }\end{array}$ & Nunununia & Solanaceae & Herb & Edible & $\begin{array}{l}\text { Fruits are edible as } \\
\text { vegetables }\end{array}$ & Present study \\
\hline $\begin{array}{l}\text { Syzgium cumini. } \\
\text { (L.) Skeels }\end{array}$ & Jamu & Myrtaceae & Tree & Edible & Fruits are edible & Present study \\
\hline $\begin{array}{l}\text { Tamarindus } \\
\text { indica } \mathrm{L} \text {. }\end{array}$ & Tentuli & Caesalpinaceae & Tree & Edible & Fruits are edible & Present study \\
\hline Zea mays $\mathrm{L}$. & Mokka & Poaceae & Grass & Edible & Seeds are edible & Present study \\
\hline $\begin{array}{l}\text { Ziziphus } \\
\text { mauritiana Lam. }\end{array}$ & Jhar koli & Rhamnaceae & Shrub & Edible & Fruits are edible & {$[8]$} \\
\hline
\end{tabular}


Table 3: List of socio-cultural, economic, and ornamental plants and common weeds in the City of Rourkela, Odisha, India

\begin{tabular}{|c|c|c|c|c|c|}
\hline $\begin{array}{l}\text { Botanical } \\
\text { names }\end{array}$ & $\begin{array}{l}\text { Local name }(\mathbf{s}) / \\
\text { Common } \\
\text { name(s) }\end{array}$ & Family & Habitat & Group(s) & $\begin{array}{l}\text { Common uses / } \\
\text { other } \\
\text { character(s) }\end{array}$ \\
\hline $\begin{array}{l}\text { Allamanda } \\
\text { cathartica } \mathrm{L} .\end{array}$ & Golden trumpet & Apocynaceae & Shrub & Ornamental & $\begin{array}{l}\text { Flowers are used Present study } \\
\text { as ornament }\end{array}$ \\
\hline $\begin{array}{l}\text { Alstonia } \\
\text { scholaris } \\
\text { R.Br. }\end{array}$ & Chatiana & Apocynaceae & Tree & Ornamental & $\begin{array}{l}\text { Planted on Present study } \\
\text { roadside } \\
\text { reduce pollution }\end{array}$ \\
\hline $\begin{array}{l}\text { Bambusa } \\
\text { vulgaris } \\
\text { Schrad. }\end{array}$ & Sundrogai & Poaceae & Grass & Ornamental & $\begin{array}{l}\text { Planted } \quad \text { for }[52] \\
\text { ornamental } \\
\text { purposes }\end{array}$ \\
\hline $\begin{array}{l}\text { Bauhinia } \\
\text { acuminate L. }\end{array}$ & Kanchanar & Caesalpinaceae & $\begin{array}{l}\text { Shrub/ } \\
\text { moderate } \\
\text { size tree }\end{array}$ & Ornamental & $\begin{array}{l}\text { Planted } \\
\text { ornamental } \\
\text { purposes }\end{array}$ \\
\hline $\begin{array}{l}\text { Bauhinia } \\
\text { purpurea } \mathrm{L} .\end{array}$ & Kuliari & Caesalpinaceae & Tree & Ornamental & $\begin{array}{l}\text { Planted } \\
\text { ornamental } \\
\text { purposes }\end{array}$ \\
\hline $\begin{array}{l}\text { Callistemon } \\
\text { citrinus stapf. }\end{array}$ & Bottlebrush & Myrtaceae & $\begin{array}{l}\text { Large } \\
\text { shrub }\end{array}$ & Ornamental & $\begin{array}{l}\text { Planted } \\
\text { ornamental } \\
\text { purposes }\end{array}$ \\
\hline $\begin{array}{l}\text { Calophyllum } \\
\text { inophyllum L. }\end{array}$ & Polang & Cluslaceae & Tree & Ornamental & $\begin{array}{l}\text { Planted } \\
\text { ornamental } \\
\text { purposes }\end{array}$ \\
\hline $\begin{array}{l}\text { Canna indica } \\
\mathrm{L} \text {. }\end{array}$ & Sarbajaya & Marantaceae & Shrub & Ornamental & $\begin{array}{l}\text { Planted } \\
\text { ornamental } \\
\text { purposes }\end{array}$ \\
\hline $\begin{array}{l}\text { Cassia siamea } \\
\text { Lam. }\end{array}$ & Seemia & Caesalpinaceae & Tree & Ornamental & $\begin{array}{l}\text { Planted } \\
\text { ornamental } \\
\text { purposes }\end{array}$ \\
\hline $\begin{array}{l}\text { Casurina } \\
\text { aquisetifolia } \mathrm{L} .\end{array}$ & & Casuarinaceae & Tree & Ornamental & $\begin{array}{l}\text { Planted } \\
\text { ornamental } \\
\text { purposes }\end{array}$ \\
\hline $\begin{array}{l}\text { Catharanthus } \\
\text { roseus (L.) G. } \\
\text { Don. }\end{array}$ & Sadabahari & Apocynaceae & Herb & Ornamental & $\begin{array}{l}\text { Planted } \\
\text { ornamental } \\
\text { purposes }\end{array}$ \\
\hline $\begin{array}{l}\text { Ceiba } \\
\text { pentandra (L.) } \\
\text { gacrtn. Fruct. }\end{array}$ & Kapok & Malvaceae & Tree & Ornamental & $\begin{array}{l}\text { Planted for Present study } \\
\text { ornamental } \\
\text { purposes }\end{array}$ \\
\hline $\begin{array}{l}\text { Celosia } \\
\text { argentea } \mathrm{L} .\end{array}$ & Sirali & Amaranthaceae & Grass & Weeds & $\begin{array}{l}\text { Very attractive Present study } \\
\text { white flowers } \\
\text { make it an } \\
\text { ornamental weed } \\
\text { in grassland }\end{array}$ \\
\hline $\begin{array}{l}\text { Cleome } \\
\text { monophylla } \mathrm{L} .\end{array}$ & Rango Sorisho & Capparaceae & Herb & Weeds & $\begin{array}{l}\text { Weed on waste Present study } \\
\text { land }\end{array}$ \\
\hline $\begin{array}{l}\text { Clerodendrum } \\
\text { unfortunatum } \\
\text { auct.non L. }\end{array}$ & Buhasin & Verbenaceae & Shrub & Weeds & $\begin{array}{l}\text { Weeds } \\
\text { roadside } \\
\text { attractive } \\
\text { with } \\
\text { flowers }\end{array}$ \\
\hline $\begin{array}{l}\text { Coccinia } \\
\text { grandis } \\
\text { Voigt. }\end{array}$ & Banakundri & Cucurbitaceae & Climber & Weeds & $\begin{array}{l}\text { Fruits are edible Present study } \\
\text { for birds }\end{array}$ \\
\hline $\begin{array}{l}\text { Corchorus } \\
\text { acutangula } \\
\text { Lam. }\end{array}$ & Indian mallow & Tiliaceae & Herb & Weed & $\begin{array}{l}\text { Insects } \quad \text { are Present study } \\
\text { attracted } \quad \text { by } \\
\text { attractive yellow } \\
\text { flowers }\end{array}$ \\
\hline
\end{tabular}


Diversity, ethnobotany of plant species of Steel City, India

\begin{tabular}{|c|c|c|c|c|c|c|}
\hline $\begin{array}{l}\text { Croton } \\
\text { bonplandianus } \\
\text { Baill. }\end{array}$ & Ban mirchi & Euphorbiaceae & Herb & Weeds & $\begin{array}{l}\text { Fruits and leaves } \\
\text { are edible for } \\
\text { Tectoris } \text { species }\end{array}$ & $\begin{array}{l}\text { Present study } \\
\text { for }\end{array}$ \\
\hline $\begin{array}{l}\text { Cycas rumphi } \\
\text { Miq. }\end{array}$ & $i$ Rosaimaro & Cycadaceae & Fern & Ornamental & $\begin{array}{l}\text { Planted fo } \\
\text { ornamental } \\
\text { purposes }\end{array}$ & for Present study \\
\hline $\begin{array}{l}\text { Cynodon } \\
\text { dactylon } \\
\text { Pers. }\end{array}$ & Dubo & Poaceae & Grass & Ornamental & $\begin{array}{l}\text { Planted fo } \\
\text { ornamental } \\
\text { purposes }\end{array}$ & for Present study \\
\hline $\begin{array}{l}\text { Cyperus } \\
\text { puneticulatus } \\
\text { Vahl. }\end{array}$ & Nutsedge & Cyperaceae & Sedges & Weed & $\begin{array}{l}\text { This is ar } \\
\text { ornamental weed }\end{array}$ & an [54] \\
\hline $\begin{array}{l}\text { Cyperus } \\
\text { rotundus } \mathrm{L} .\end{array}$ & Motha & Cyperaceae & Sedges & Weed & $\begin{array}{l}\text { This is ar } \\
\text { ornamental weed }\end{array}$ & an [54] \\
\hline $\begin{array}{l}\text { Cyperus triceps } \\
\text { Endl. }\end{array}$ & Flatsedge & Cyperaceae & Sedges & Weed & $\begin{array}{l}\text { This is ar } \\
\text { ornamental weed }\end{array}$ & an [54] \\
\hline $\begin{array}{l}\text { Dalbergia } \\
\text { sissoo Roxb. }\end{array}$ & Sissoo & Fabaceae & Tree & Timber & $\begin{array}{l}\text { Wood is used as } \\
\text { timber }\end{array}$ & Present study \\
\hline $\begin{array}{lr}\text { Delonix } & \text { regia } \\
\text { (Bojex } & \text { Hook.) } \\
\text { Raf. } & \end{array}$ & a Gulmohar & Caesalpinaceae & Tree & Ornamental & $\begin{array}{l}\text { Planted fo } \\
\text { ornamental } \\
\text { purposes }\end{array}$ & for Present study \\
\hline $\begin{array}{l}\text { Desmodium } \\
\text { triflorum } \\
\text { DC. }\end{array}$ & Kuradia & Fabaceae & Herb & Ornamental & $\begin{array}{l}\text { Planted } \\
\text { ornamental } \\
\text { purposes }\end{array}$ & for Present study \\
\hline $\begin{array}{l}\text { Drypetes } \\
\text { roxburghii } \\
\text { Wall. }\end{array}$ & Poisundia & Euphorbiaceae & Climber & Ornamental & $\begin{array}{l}\text { Planted } \\
\text { ornamental } \\
\text { purposes }\end{array}$ & for Present study \\
\hline $\begin{array}{l}\text { Duranta repens } \\
\text { L. }\end{array}$ & $\begin{array}{l}\text { Golden } \\
\text { dewdrop }\end{array}$ & Verbenaceae & Shrub & Ornamental & $\begin{array}{l}\text { Planted } \\
\text { ornamental } \\
\text { purposes }\end{array}$ & for Present study \\
\hline $\begin{array}{l}\text { Eichhornia } \\
\text { crassipes } \\
\text { (Mart.) Solms. }\end{array}$ & Water-Hyacinth & Pontederiaceae & Herb & Weed & $\begin{array}{l}\text { Not good } \\
\text { water bodies }\end{array}$ & for Present study \\
\hline $\begin{array}{l}\text { Eucalyptus } \\
\text { tereticornis } \mathrm{Sm} \text {. }\end{array}$ & Eucalptus & Myrtaceae & Tree & Timber & $\begin{array}{l}\text { Wood is used as } \\
\text { timber }\end{array}$ & Present study \\
\hline $\begin{array}{l}\text { Euphorbia milii } \\
\text { Des. Moul. }\end{array}$ & Crown of thorns & Euphorbiaceae & Herb & Ornamental & $\begin{array}{l}\text { Planted fo } \\
\text { ornamental } \\
\text { purposes }\end{array}$ & for Present study \\
\hline $\begin{array}{lr}\text { Ficus } & \text { elastic } \\
\text { Roxb. } & \text { ex } \\
\text { Hornem. } & \end{array}$ & $c$ Rubber tree & Moraceae & Tree & Ornamental & $\begin{array}{l}\text { Planted fo } \\
\text { ornamental } \\
\text { purposes }\end{array}$ & for Present study \\
\hline $\begin{array}{l}\text { Hedyotic } \\
\text { corymbosa (L.) } \\
\text { Lam. }\end{array}$ & Dimond Flower & Rubiaceae & Herb & Weed & $\begin{array}{l}\text { Common weed, } \\
\text { everywhere } \\
\text { city }\end{array}$ & $\begin{array}{l}\text { Present study } \\
\text { in }\end{array}$ \\
\hline $\begin{array}{l}\text { Heliotropium } \\
\text { supinum L. }\end{array}$ & $\begin{array}{l}\text { Dwarf } \\
\text { Heliotrope }\end{array}$ & Boraginaceae & Herb & Weed & $\begin{array}{l}\text { Common } \\
\text { shady areas } \\
\text { the city }\end{array}$ & $\begin{array}{l}\text { in Present study } \\
\text { of }\end{array}$ \\
\hline $\begin{array}{l}\text { Hibiscus } \\
\text { sinensis L. }\end{array}$ & - Mandara & Malvaceae & Shrub & Ornamental & $\begin{array}{l}\text { Flowers are used } \\
\text { for worship }\end{array}$ & Present study \\
\hline $\begin{array}{l}\text { Ipomea carnea } \\
\text { Jacq. }\end{array}$ & a Omori & Convolvulaceae & Shrub & Weed & Toxic to animals & Present study \\
\hline $\begin{array}{l}\text { Ipomea } \\
\text { Haines }\end{array}$ & $e$ Irit- Irit & Convolvulaceae & Climber & Weed & $\begin{array}{l}\text { Common climber } \\
\text { on walls }\end{array}$ & Present study \\
\hline $\begin{array}{l}\text { Ipomea } \\
\text { sepiaria }\end{array}$ & Bilona & Convolvulaceae & Climber & Weed & $\begin{array}{l}\text { Common climber } \\
\text { on walls }\end{array}$ & Present study \\
\hline Koeing & & & & & & \\
\hline
\end{tabular}


Roxb.

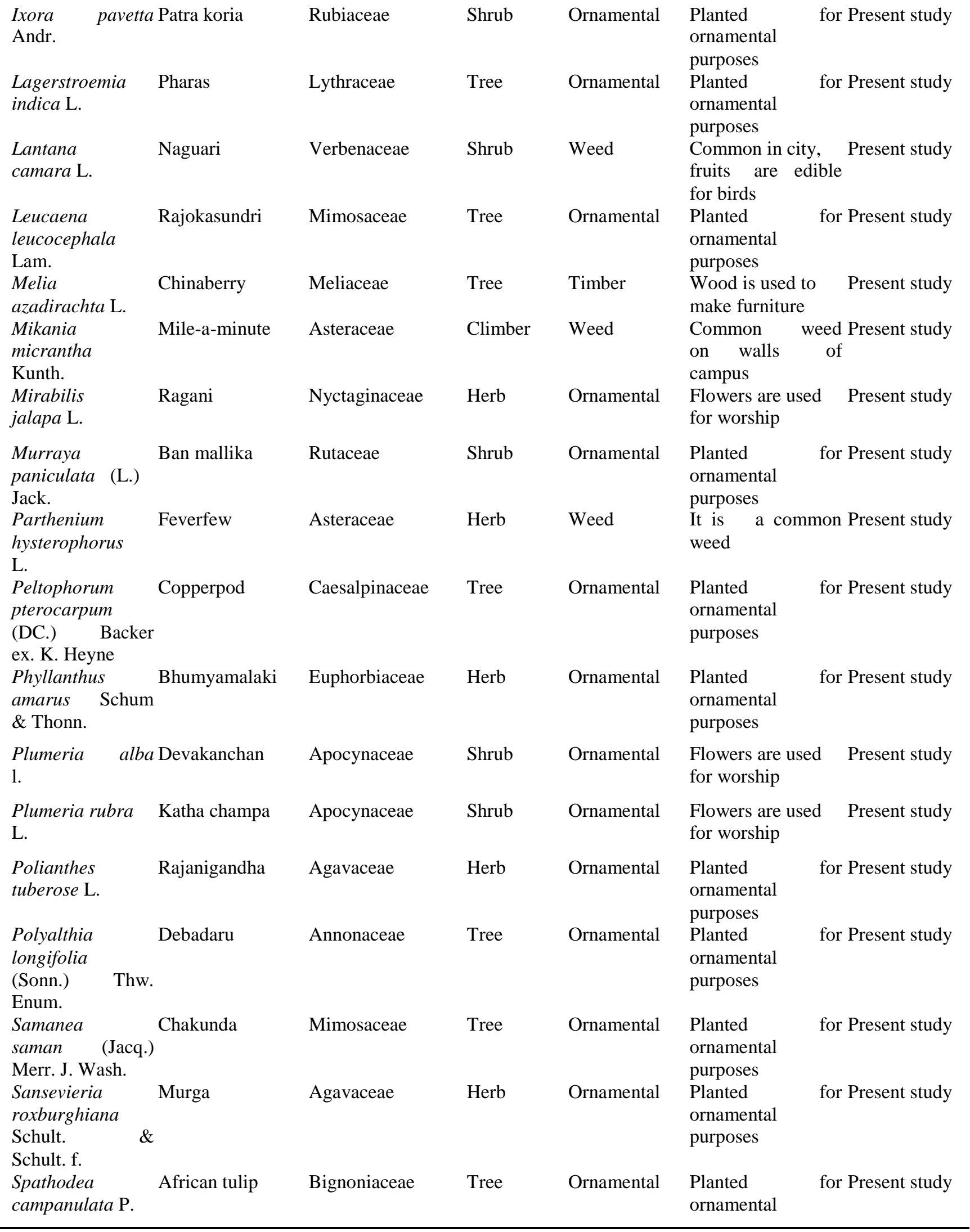


Diversity, ethnobotany of plant species of Steel City, India

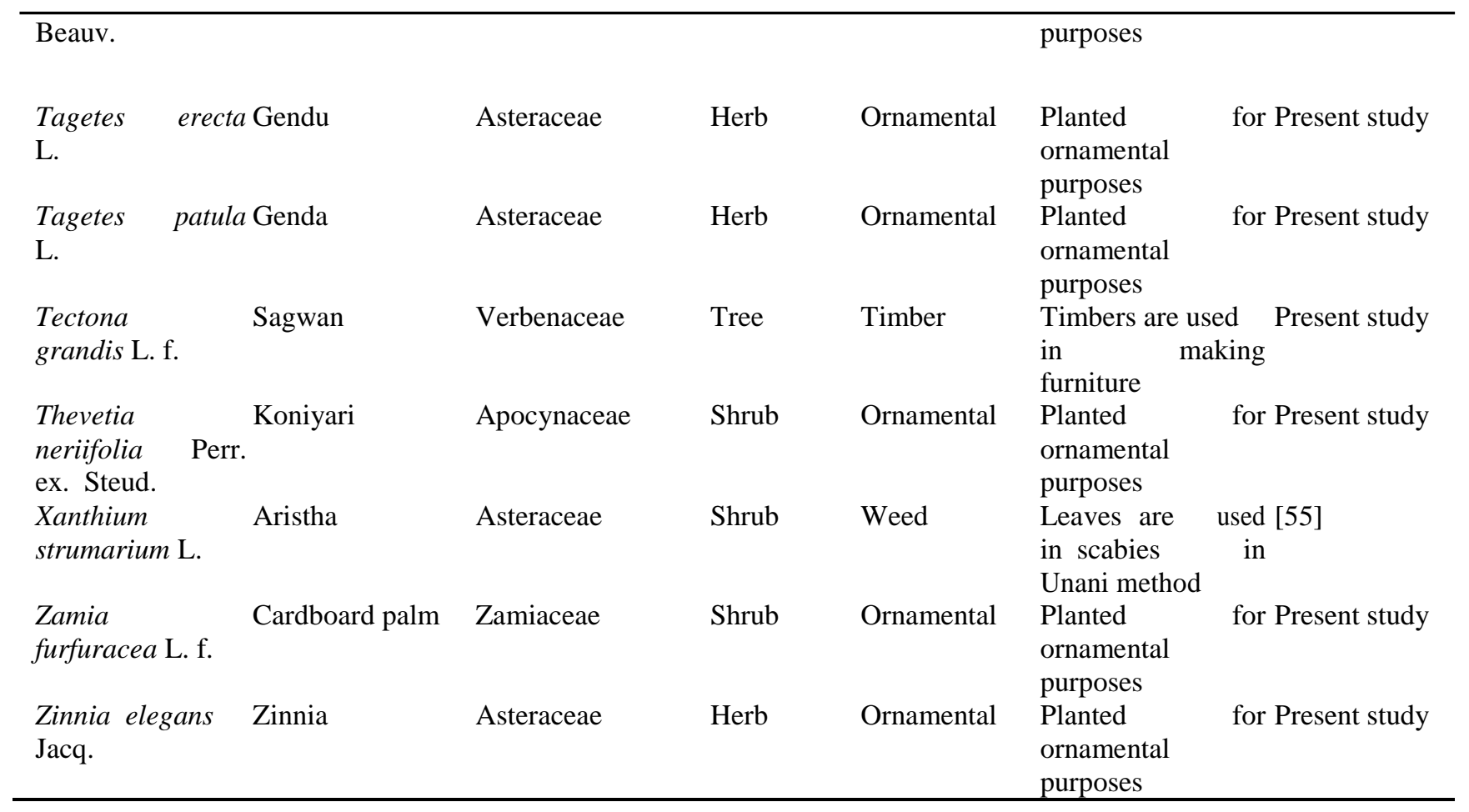

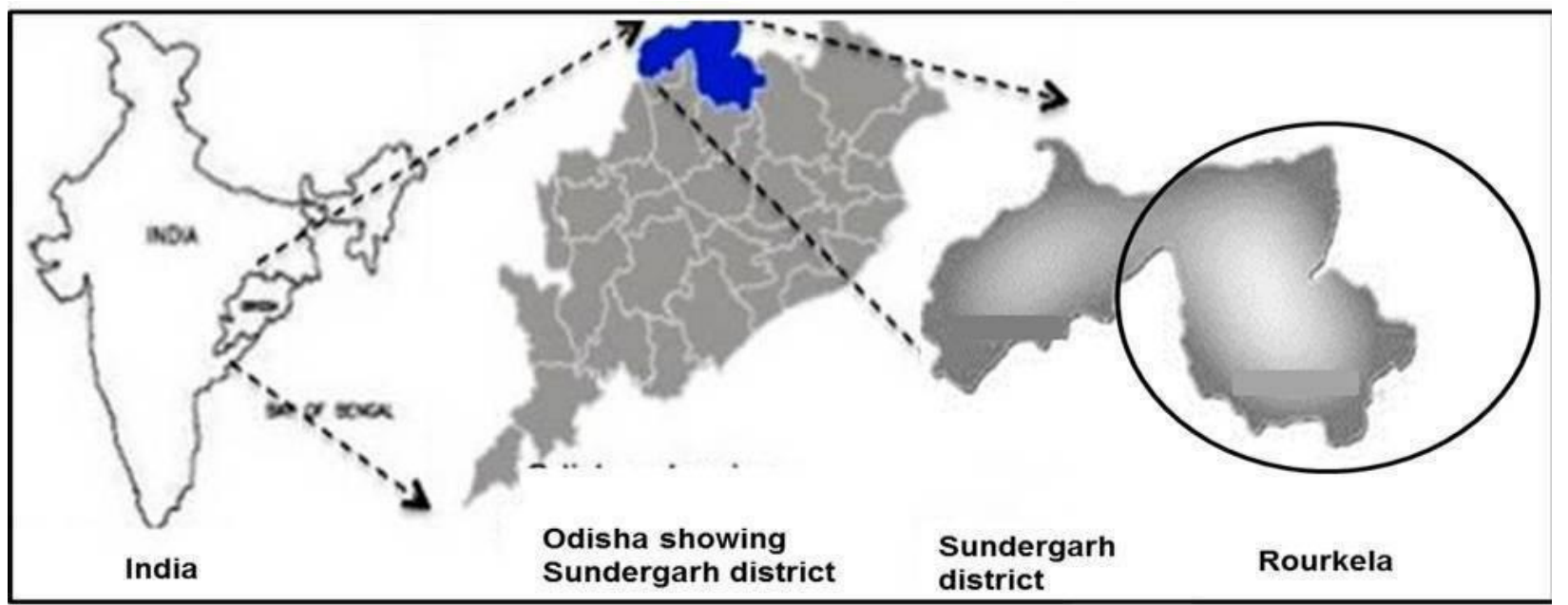

Plate-1: Geographical location of the study area 


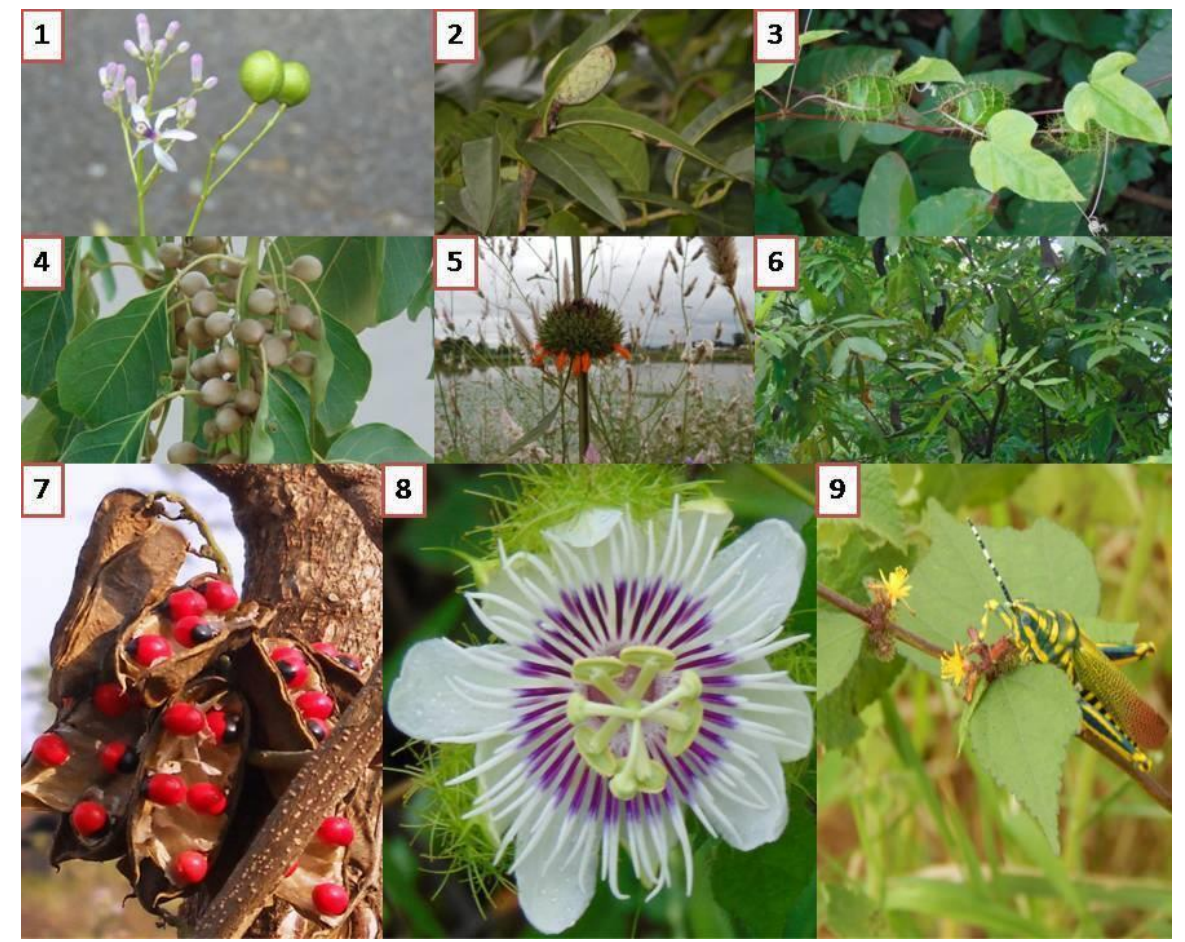

Plate 2: Photos showing selected plant species from Rourkela city, Odisha, India,

1) Flowers and fruits of Melia azadirachta L., 2) Fruit of Annona reticulata L., 3) Fruits of Passiflora foetida L., 4) Fruits of Terminalia bellirica (Gaertn.) Roxb., 5) Flowers of Leonotis nepetifolia (L.) R.Br., 6) Fruits and leaves of Saraca asoca (Roxb.) Willd., 7) Seeds of Abrus precatorius L., 8) Flower of Passiflora foetida L., 9) Triumfetta pentandra A. Rich.

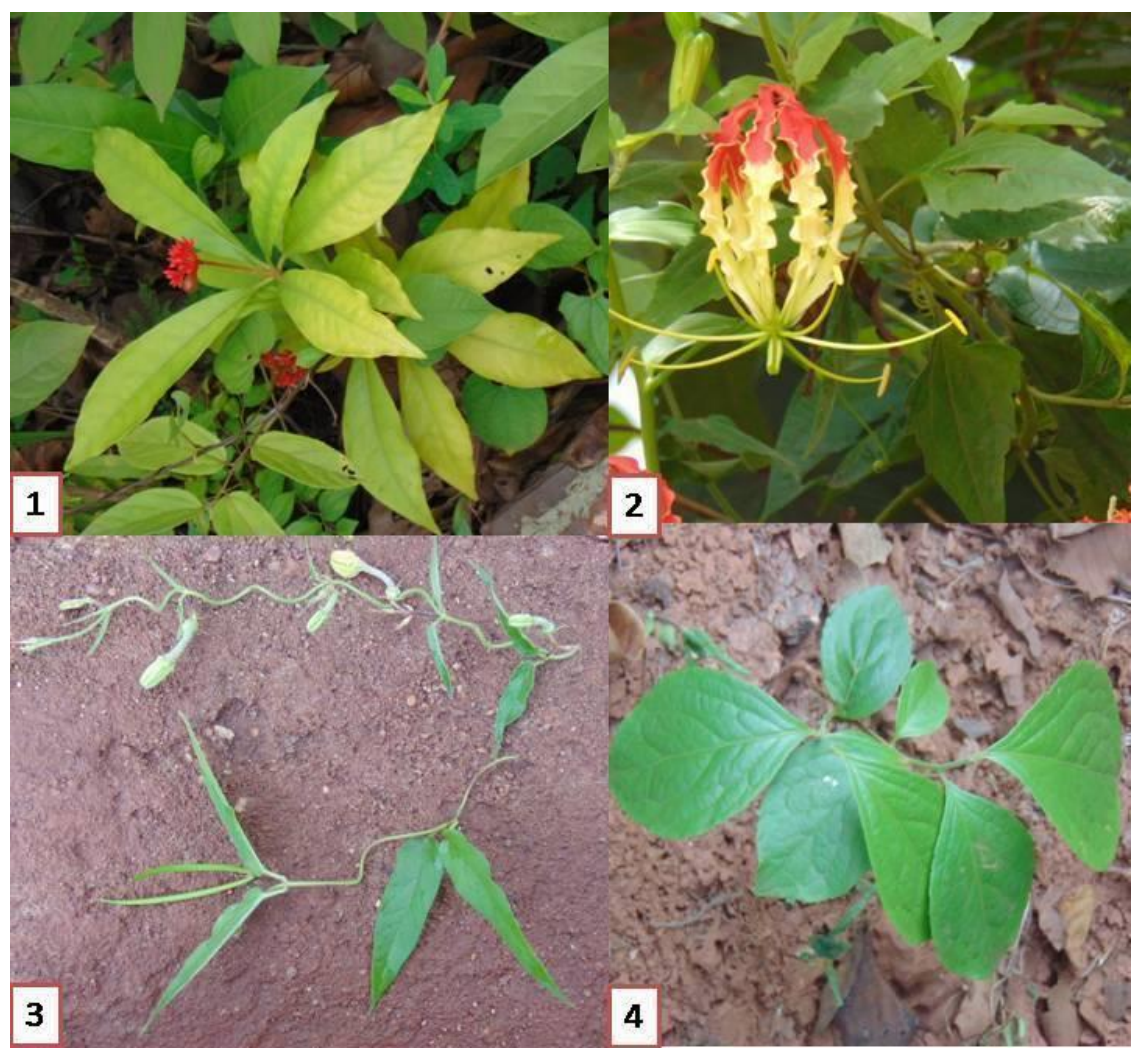

Plate 3: Some RET plant species of the district Sundargarh (Rourkela city is situated in this district), Odisha, India,

1) flowers of Rauvolfia serpentina; 2) Flowers of Gloriosa superba; 3) Flowers of Ceropegia hirsuta*;

4) Plantlet of Celastrus paniculatus. *Authors found very less population in the state. 
Diversity, ethnobotany of plant species of Steel City, India

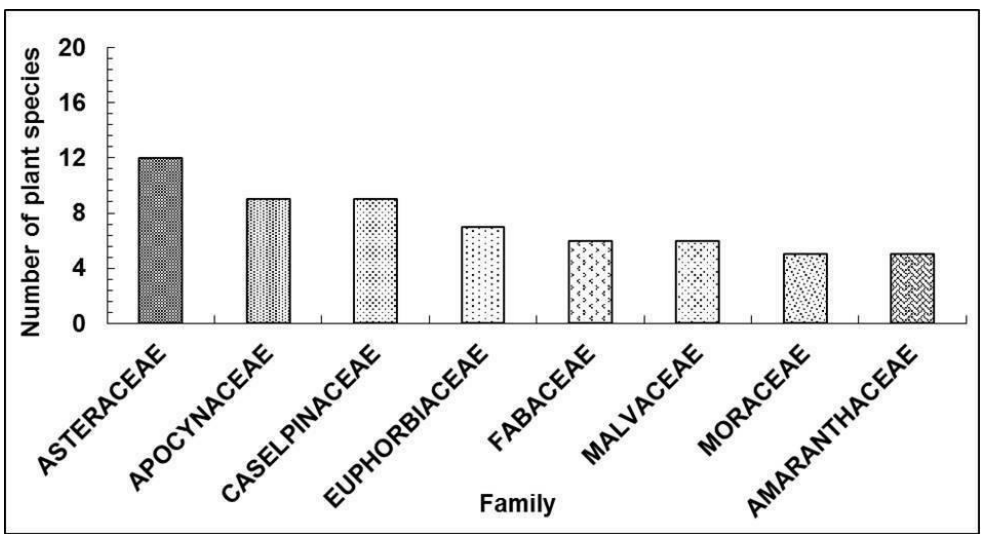

Figure 1. The eight dominant families in city of Rourkela, Odisha, India.

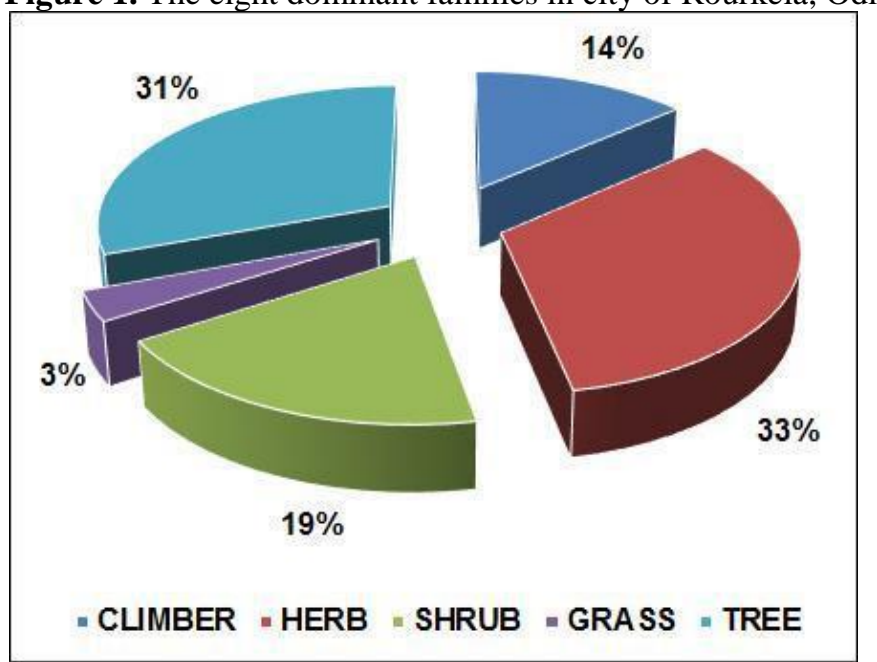

Figure 2: Diversity of plant species by habitat.

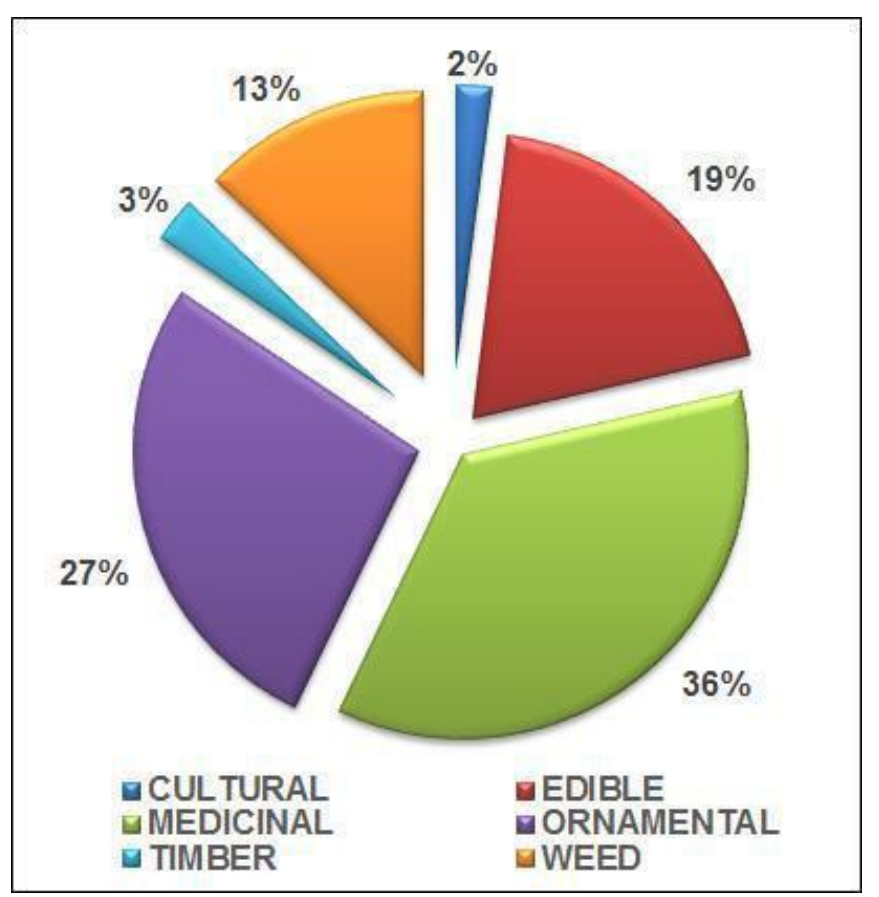

Figure 3: Plants used for different purposes. 


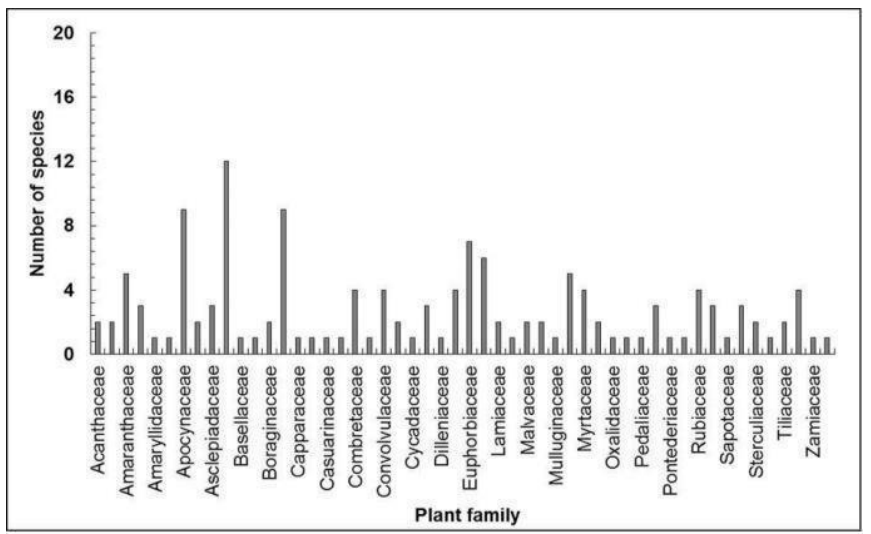

Figure 4: Number of plant species in each family.

\section{DISCUSSION}

Traditional knowledge and the use of local plants for primary needs remain important, not only in rural or tribal areas but even in urban and semi-urban areas. The collected information on the useful plants in and around Rourkela city, Odisha, India, includes some that are medicinal, some that are edible, and some that are socio-cultural plants. As therapeutics, the hitherto unknown claims from local people, such as use of the root and bark of Abutilon indicum as nervine tonic, of the leaves of Paderia foetida against diarrhea were documented (Table 1), of the flowers of Saraca asoka (Plate 2.6) in painful menses, and of the leaves of Datura metel to cure different types of fungal infections. Of the edible plants, the fruits of Aegle marnelos are consumed in summer as a cooling agent (Table 2), the leaves of Basella alba are used as leafy vegetables in a delicious local dish, the tubers of Dioscorea bulbifera are eaten as vegetables (Table 2) after successive boiling owing to the presence of some unsavory factors such as tannin, the fruit of Emblica officinalis is used to make a medicinal pickle, and the leaves of Murraya koenigii are used to give aroma to vegetables and other foods of the state, etc. (Present study). Very interesting socio-cultural and economic plants are available in the city, such as the flowers of Plumeria alba, Hibiscus rosa-sinensis, and Mirabilis jalapa (Table 3) that are used during worship, and the timber of Tectona grandis, Eucalyptus tereticornis, and Melia azadirachta (Plate 2.1) that are used in making furniture etc. (Present study).

Globally, the bio-resources of some urban areas with a view to conservation have been reported by various researchers. Ma and Liu [56] have reported about 455 plants in the urban area of Beijing, China, whereas Grdovic and Stevanovic [57] have documented 58 plant species in the central urban area of Belgrade, Serbia. Thompson and McCarthy [58] have reported 822 vascular plants in the cities of Sheffield and Birmingham, in the United Kingdom. Muratet et al. [59] have documented 626 vascular plants in the urban area of the Hauts-de-Seine district bordering Paris, France, while Yavorska [60] has reported about 114 plant species in the urban area of Kyiv, Ukraine. Pieniak and Chylinski [61] have reported 26 spontaneously-occurring floras in industrial buildings of Warsaw, Poland. Recently, Goraya et al. [62] have reported about 60 plant species with medicinal value in the peri-urban areas of Punjab in Pakistan. Many domestic researchers have likewise reported on urban flora and its ethnomedicinal importance. Singh [63] has reported around 765 medicinal plant species in the urban area of Varanasi, India. Kumar and Satapathy [30] have reported a total of 72 herbaceous plants in the urban area of Bhubaneshwar, India. Singh [64] has reported around 119 vascular plants at Banaras Hindu University, Varanasi, India. Verma et al. [21] have reported about 72 
Diversity, ethnobotany of plant species of Steel City, India

medicinal plants on the campus of Banaras Hindu University, Varanasi, India. Recently, Pasayat et al. [25] have reported about 20 ethno-toxic effects of some common angiosperms found around Rourkela city, India.

The present study revealed that most of the plant species belong to the asteraceae and apocynacea families and showed a rich diversity of useful wild and cultivated species with potential ethnomedicinal value. However, in contrast to the above findings, Mallick et al. [65] have reported that the city enjoys a flora of asteraceae and poaceae and most of the plants are weeds. Though the grasses that belong to the family asteraceae and poaceae are dominant in the urban area, but there are many other plants found in this area which are not weeds, they are wild in nature with prominent medicinal values. Gradually, when these area developed to an urban area, the wild plants available in these areas were later on termed as the urban floras but not as weeds. In 2013, Pasayat et al. [25] reported about 20 ethno-toxic plant species from the present study area. Among these species, they claimed that parts of Datura metal L. are toxic, whereas the present study focused on ethnomedicinal properties and found that all plant parts are very much effective against all types of skin infections (Table 1), while the flowers have socio-cultural and economic value and are sold for worship outside Hindu temples around India. In 2014, Mallick et al. [26] documented 30 ethnomedicinal shrubs in the city of Rourkela, India. They reported that the leaves of Paederia foetida L. are used against joint pain, gastric problems, and diarrhea, while the present study has found that this is a very important ethnomedicinal plant of the city as it falls in the RET (rare, endangered and threatened) group and the leaves are frequently used in urban communities in the study area against diarrhea (Table 1). The present study supports the claim that seed oil of Ricinus communis $\mathrm{L}$. is used to cure skin infections and is also effective against joint pain [26]. Ives et al. [66] reported that cities are the hotspots of RET (rare, endangered and threatened) plant species. The forest patches of the district is rich with many RET plant species (Plate 3). Among them, present study also suggests that the city is home to two RET plants (Saraca asoka and Paederia foetida) of the state and an ongoing study addresses the sustainable harvesting of these two useful plants of the Steel City of Rourkela, Odisha, India. Many other plant species found in this area are of potential medicinal importance.

\section{CONCLUSION}

Urbanization has increased tremendously over the last 60 years around the world, with the result that more than 50 percent of the world population now live in cities. This is especially true for developing countries, and it is expected that developing countries will take the lead in future urban population growth. Increasing urbanization has serious consequences for the environment, as it fragments and changes natural habitats and alters environmental conditions. Therefore, there is a need for more exploration and awareness of research with the aim of conserving urban floras. It is has also emerged quite recently that not only natural and seminatural landscapes can be highly diverse in terms of flora, but that urban, institutional and industrial areas may also harbor a wide variety of habitats, organisms, and communities. The use of local floras as primary medical resources and traditional practices of plant-based medico-foods are still alive in the modern urban or semi-urban areas such as Steel City (Rourkela) of the State of Odisha, India. The present study illustrates to what extent urban communities may depend on various plants to meet their needs and to cure various diseases and disorders by means of traditional medicines. Appropriate conservation planning is therefore 
required to conserve these useful floras and to maintain biodiversity in this urban area, which underpins traditional knowledge.

\section{ACKNOWLEDGEMENTS}

The authors are grateful to the local people around the Rourkela city, Odisha, India for their support and cooperation during field surveys and for providing information on the medicinal potential of the plant species. This work was supported by Korea Institute of Planning and Evaluation for Technology in Food, Agriculture, Forestry and Fisheries (IPET) through the Agricultural Research Center Project and Agricultural Bio-Technology Development Program funded by Ministry of Agriculture, Food and Rural Affairs(MAFRA)(710003-07-7-SB120, 116075-3).

\section{REFERENCES}

1. Tilman D, Lehman C. Human caused environmental change: Impacts on plant diversity and evolution. Proceed. Nat Acad Sci United States Amer. 2001; 98: 5433-5440.

2. Maity D, Pardhan N, Chauhan AS. Folk uses of some medicinal plants from North Sikkim. Ind J Trad Know. 2004; 3: 66-71.

3. Alves RRN, Rosa IML. Biodiversity, traditional medicine and public health: where do they meet. J Ethno Ethnomed. 2007; 3: 1-14.

4. Sedelnikov VP, Naumenko YV, Sedelnikova NV, Gorbunova IA, Pisarenko OY, Shaulo DN. Biodiversity and spatial arrangement of Siberian flora. Contemp Problem Ecol. 2008; 1: 14-21.

5. Panskus AB, Breckling B, Hamberger S. Cultivation-independent establishment of genetically engineered plants in natural populations: current evidence and implications for EU regulation. Environ Sci. Europ. 2013;25:34-35.

6. Akpabio UD, Ikpe EE. Proximate composition and nutrient analysis of Aneilema aequinoctiale leaves. Asian J Plant Sci Rese. 2013; 3: 55-61.

7. Dahlberg AC, Trygger SB. Indigenous medicine and primary health care: The importance of lay knowledge and use of medicinal plants in rural South Africa. Hum Ecol. 2009; 37: 79-94.

8. Kumar S, Dash D. 2012. Flora of Nandan Kanan Sanctuary: medicinal plants with their role in health care. Int J Pharm Life Sci. 2013; 3: 1631-1642.

9. Sen S, Chakraborty R, De B, Devanna N. 2011. An ethanobotanical survey of medicinal plants used by ethnic people in west and south district of Tripura, India. J Forest Res. 2011; 22: 417-426.

10. Sahu CR, Nayak RK, Dhal NK. The plant wealth of Boudh district of Odisha, India with reference to ethanobotany. Int J Curr Biot. 2013; 1: 4-10.

11. Aliotta G, Mallik UM, Pollio A. 2008. Historical Examples of Allelopathy and Ethanobotany from the Mediterranean Region. Allelo Sust Agri Forest. 2008; 1: 11-24.

12. Tizio AD, Luczaj LJ, Quave CL, Redzic S, Pieroni A. Traditional food and herbal uses of wild plants in the ancient South-Slavic diaspora of Mundimitar/Montemitro(Southern Italy). J Ethano Ethnomed. 2012;8:21-21.

13. Posey DA, Dutfield G. Beyond Intellectual property: toward traditional resource rights for Indigenous people and local communities, Canada. Int Develop Res 1996; 303: 1-10.

14. Catarino L, Havik PJ, Romeiras MM. Medicinal plants of Guinea-Bissau: therapeutic applications, ethnic diversity and knowledge transfer. Ethnopharmacol. 2016; 183:71-94.

15. Sadeghi Z, Mahmood A. Ethno-gynecological knowledge of medicinal plants used by Baluch tribes, southeast of Baluchistan, Iran. Revi Brasilei de Farmacogno. 2014; 24(6): 706-715.

16. Chaudhary S, Kumar R. Some important medicinal trees of district Bijnor. Rece Res Sci Tech. 2011;3:96-100.

17. Banarjee A, Mukherjee A, Sinhababu A. Ethanobotanical documentation of some wild edible plants in Bankura district, West Bengal, India. J Ethan Trad Med. 2013; 120: 585590. 
Diversity, ethnobotany of plant species of Steel City, India

18. Singh A. Vascular floristic wealth of Banaras Hindu University main campus, India. Int J Plant Sci. 2013a;2:124-149.

19. Padal SB, Sandhyasri B. Ethanomedicinal investigation of medicinal plants of Sovva panchayat, Dumbriguda Mandalam, Visakhapatanam district, Andhra Pradesh. Int J Eng Sci. 2013; 2: 55-61.

20. Mohmood A, Qureshi RA, Mahmood A, Sangi Y, Shaheen H, Ahmad I, Nawaz Z. Ethnobotanical survey of common medicinal plants used by people of district Mirpur, AJK. Pak J Med Plants Res. 2011; 5: 4493-4498.

21. Verma AK, Kumar M, Bussmann RW.Medicinal plants in an urban environment: the medicinal flora of Banaras Hindu University, Uttar Pradesh. J Ethn Ethnomed. 2007; 3: 35-35.

22. Saxena N, Yadav VK, Verma RK. Traditional knowledge of medicinal plants used to cure gastro intestinal problems in Jalan district of Utter Pradesh, India. J Med Plant Stud. 2014; $2: 24-28$.

23. Prusti AB, Behera KK. Ethno-Medico Botanical study of Sundargarh district, Orissa, India. Ethnobot Leaf. 2007;11:148-163.

24. Acharya BC, Subudhi HN, Panda SP. Check list of economic plants of Rourkela and adjoining regions (Sundargarh district)- Odisha. Bull Pure App Sci Bot. 2010; 29: 53-57.

25. Pasayat SK, Sahoo P, Mallick SN. Ethno-toxic effects of some common angiosperms plants studies in and around Rourkela, Sundargarh, Odisha. Life Sci Leaf. 2013;4:12-15.

26. Mallick SN, Ram JP, Parida N. Study of ethnomedicinal values of some shrub in Rourkela steel city and its surroundings, Sundergarh, Odisha. Int J Appl Biol Pharm Tech. 2014; 5: 123-130.

27. Christian RV, Brigitte VL. Tools and methods for data collection in ethnobotanical studies of homegardens. Field Method. 2004;16:285-306.

28. Haines HH. The Botany of Bihar and Orissa. Adlard \& Sons and West Newman Ltd; London; 1921.

29. Saxena HO, Brahmam M. Flora of Orissa. Orissa Forest Development Corporation \& Regional Research Laboratory; Bhubaneswar; 1994.

30. Kumar S, Satapathy MK. Medicinal plants in an urban environment; herbaceous medicinal flora from the campus of regional Institute of Education, Bhubaneswar, Odisha. Int J Pharma Life Sci. 2011; 2: 1206-1210.

31. Khadabadi SS, Bhajipale NS. A review on some important medicinal plants of Abutilon spp. Res J Pharmac Biol Chem Sci. 2010; 1: 718-729.

32. Panda A, Misra MK. Ethnobotanical survey of some wetland plants of South Orissa and their conservation. Ind J Trad Know. 2011; 10: 296-303.

33. Thirunavoukkarasu M, Balaji U, Behera S, Panda PK, Mishra BK. Biosynthesis of silver nanoparticle from leaf extract of Desmodium gangeticum (L.) DC. and its biomedical potential. Spect Acta A Mol Biomol Spect. 2013; 116: 424-427.

34. Chokotia S, Vashistha P, Sironiya R, Matoli H. Pharmacological activities of Eclipta alba (L.). Res Develop Pharma Life Sci. 2013; 2: 499-502.

35. Mohan VR, Chenthurpandy P, Kalidass C. Pharmacognostic and phytochemical investigation of Elephantopus scaber L. (Asteraceae). J Pharma Sci Tech. 2010; 2: 191197.

36. Essien GE, Nwidu LL, Nwafor PA. Anti-Inflammatory and Analgesic Potential of Methanolic Extract of Emilia Sonchifolia (Compositae) Leaves in Rodents. African J Biomed Res. 2009; 12: 199-207.

37. Ahmad SF, Khan B, Bani S, Kaul A, Sultan P, Ali SA, Satti NK, Bakheet SA, Sabry MA, Khairy MA, Allah ARA. (2012). Immunosuppressive effects of Euphorbia hirta in experimental Animals. Inflammophar, http: //dx.doi.org. 10.1007/s10787-012-0144-6.

38. Rao N, Kaladhar D. Antioxidant and antimicrobial activities of rhizome extracts of kaempferia galangal. World J Pharma Pharma Sci. 2014;3:1180-1189.werk,jjul im

39. Ali M, Chaudhary N. Ficus hispidaLinn.: A review of its pharmacognostic and ethnomedicinal properties. Pharmacohn Rev. 2011; 5: 96-102.

40. Patil JU, Birdar SD. Pharmacognostic study of Guazuma ulmifolia. Int Res J Pharm. 2013; 4: 130-131. 
41. Irobi ON, Daramola SO. Bactericidal properties of crude extracts of Mitracarpus villosus. J Ethnopharmacol.1994; 42: 39-43.

42. Chatterjee P, Chakraborty B, Nandy S, Dwivedi A, Datta R. Pterospermum acerifolium linn.: A comprehensive review with significant pharmacological activities. Int J Pharma Life Sci. 2012; 3: 1453-1458.

43. Swain SR, Sinha BN, Murthy PN. Subchronic toxicity studies of the hydroalcoholic extract of Rungia pectinata leaves. Pharmacolog. 2008; 2: 461-466.

44. Suntonvipart P, Chantachon S, Koseyayothin M. Medicinal herbs: traditional knowledge used for constipation therapy in metropolitah Bangkok. Ind J Trad Know. 2014; 13: 466472.

45. Joshi AB, Aswathi M, Bhobe M. Terminalia tomentosa Roxb (ex DC) Wight \& Arn. : Phytochemical investigation. Amer J Adv Drug Deliv. 2013; 1: 224-231.

46. Shukla AK, Mishra SP, Varma R. Anti-inflammatory, analgesic and antipyretic activities of root of Wattakaka volubilis. Int J Pharm Tech Res. 2011; 3: 1334-1338.

47. Koul S, Pandurangan A, Khosa RL. Wedelia chinenis(Asteraceae) - An overview. Asian Pac J Trop Biomed. 2012; S2: 380-383.

48. Geetha T, Komalavalli N, Subramanian SS. Antibacterial activity of the medicinal plant Wrightia tinctoria (Roxb.) R.br. Int J Pharma Bio. 2014; 5: 1137-1141.

49. Duddukuri GR, Sastry YN, Kaladhar DSGK, Rao KK, Chaitanya KK. Antibacterial activity of methanolic seed coat extract of Borassus flabellifer L. Int J Pharmac Sci Res. 2011; 2: 2435-2438.

50. Gandhi D, Mehta P. Dillenia indica Linn. andDillenia pentagyna Roxb.: pharmacognostic, phytochemical and therapeutic aspects. J App Pharma Sci. 2013; 3: 134-142.

51. Baliga MS Dsouza JJ. Amla (Emblica officinalis Gaertn.), a wonder berry in the treatment and prevention of cancer. Eur J Cancer Prev. 2011; 20: 225-239.

52. Raji R, Raveendran K. Belief and uses of Bambusa vulgaris with special refrence to Kurichya tribe of Wayanadu. Life Sci Leaf. 2011; 13: 417-442.

53. Asolker LV, Kakkar KK, Chakre OJ. $2^{\text {nd }}$ Supplement to glossary of Indian Medicinal plants. Part-I. National Institute of Sciences Communication; New Delhi; 2000.

54. Bronsnan JT, Frank JD. Purple nutsedge control in turf and ornamental. Landscap. 2008; L9: 1-4.

55. Bhogaonkar PK, Ahmad SA. Pharmacognostic studies on Xanthium strumarium: a folk Unani medicinal herb. Biosci Discov. 2010;3:101-106.

56. Ma J, Liu Q. Flora of Beijing: an overview and suggestion for future research. Urb Habit. 2003;1:30-44.

57. Grdovic S, Stevanonvic V. The moss flora in the central urban area of Belgrade. Arch Biol Sci Belg. 2006; 58: 55-59.

58. Thompson K, McCarthy MA. Traits of British alien and native urban plants. J Ecol. 2008; 96: 853-859.

59. Muratel A, Porcher E, Devictor V, Arnal G, Moret J, Wright S, Machon N. Evaluation of floristic diversity in urban areas as a basis for habitat management. Appl Veg Sci. 2008; 11: 451-460.

60. Yavorska OG. The North American species of the non-native flora of Kyiv urban area (Ukraine). Biod Res Conserv. 2009; 13: 25-30.

61. Pieniak BF, Chylinski K. Diversity of spontaneous flora of unused industrial buildings in Warsaw. Poland Ekolo Bratisal. 2012; 31: 379-389.

62. Goraya K, Iqbal Z, Sajid MS, Muhammad G, Ain QU, Saleem M. Diversity in selected per-urban areas of Punjab, Pakistan. J Ethnobiol Ethnomed. 2013; http://dx.doi.org/10.1186/1746-4269-9-70

63. Singh A, Singh MK, Singh R. Traditional medicinal flora of the district Buxar (Bihar, India). J Pharma Phytochem. 2013b; 2: 41-49.

64. Singh A. Exotic flora of Banaras Hindu University Main campus, India. J Ecol Nat Environ. 2011; 3: 337-343.

65. Mallick SN, Maharana MR, Acharya BC. Weed flora of Rourkela and adjoining areas of Sundargarh district, Odisha, India. J Eco Tax Bot. 2015; 39: 131-137. 
Diversity, ethnobotany of plant species of Steel City, India

66. Ives CD, Lentini PE, Threlfall CG, Ikin K, Shanahan DF, Garrard GE, Bekessy SA, Fuller RA, Mumaw L, Rayner L, Valentine LE, Kendal D. Cities are hotspots for threatened species. Glob Ecol Bio. 2016; 25: 117-126.

Received: February 03, 2016; Accepted: July 14, 2016 